\title{
Strukturschema und Operatoren in Hartmanns Iwein
}

\author{
Von Rolf Selbmann (München)
}

\section{ABSTRACT}

Auf der Grundlage neuerer Arbeiten zur Struktur von Hartmann von Aues Iwein soll eine gemeinsame "Interpretationsbasis" dieser Ansätze in Form eines Strukturschemas herausgearbeitet werden. Auf dieser Basis versuchen verschiedene “Operatoren” spezifische Strukturzüge des Iwein deutlicher nachzuzeichnen.

The author sets himself the task of working out a common basis of interpretation in form of a structural pattern of Hartmann von Aue's Iwein founded on recent studies. An attempt is made with a scheme of "operators" to trace specific structural features of Iwein more clearly than has been done before.

\section{Abstraktion und Strukturprinzip}

Die dialektische Struktur der Artusromane als Selbstfindung des Helden auf einem Weg mit typischen Modellstationen findet sich als Grundschema auch im Iwein. Darüber hinaus bleibt zu fragen, ob die Konfrontation von Gegensätzen und die Úberspitzung von Paradoxien ejner spezifischen Vorliebe Hartmanns entsprechen. Untersuchungsansätze dazu bieten Motivdoppelungen und -wiederholungen ${ }^{1}$ im Artusroman allgemein, die jedoch im Iwein mit fast penetranter Häufigkeit ausgespielt und abgewandelt werden. Personen (Keie/Gawein/Iwein/Kalogrenant), Dialoge (Keie/Kalogrenant, Gawein/Iwein, Lunete/Laudine usw.), Schauplätze und Aktionen sind miteinander konfrontiert, dialektische Gegenpositionen werden ins Paradoxe übersteigert (Erzählerexkurse) und wunderbare Ereignisse ins Märchenhafte aufgelöst (Löwe, Ringe, Salbe, Brunnenunwetter). Für Hartmann bedeutet das einen Lösungsversuch als Deutung eines schon von Chrestien gedeuteten Stoffes, ${ }^{2}$ nochmals kompliziert durch die Kombination von Übersetzer- und Erzählhaltung. Aus dieserı Problemen abstrahiert sich als 'literarischer Inhalt' die Emanzipation des "sen" von der "matiere" zum Zweck des Aufzeigens einer dialektischen Lösung.

1 vgl. Thomas Cramer, "'Saelde' und 'êre' in Hartmanns 'Iwein'," Euphorion, 60 (1966), 43f. - Max Wehrli, "Strukturen des mittelalterlichen Romans: Interpretationsprobleme," Formen mittelalterlicher Erzäblung (I969), S. 43 f. - Erich Köhler, Ideal und Wirklicbkeit in der böfischen Epik: Studien zur Form der früben Artus- und Graldichtung, 2. Aufl. (= Beihefte zur ZfrPh 97) (1970), S. 238.

${ }^{2} \mathrm{H}$. Burger, "Vorausdeutungen und Erzählstruktur in mittelalterlichen Texten," Festscbrift für M. Webrli (I969), S. I 50, der daraus eine "unzeitliche Struktur" ableitet. 
Schon im Stoff der Gattung Artusroman sind Extremsituationen auf dem Weg des Helden durch Abenteuer und Minne angelegt. Diese Grundstrukturen $^{3}$ komplizieren sich im Iwein, da der Text als individuelle Ausprägung auf dem Gattungstyp, Chrestiens Gattungsrezeption und dem Erec Chrestiens und Hartmanns aufliegt. Literarische Formeln und Formen können hereinzitiert werden (vgl. Gaweins Erec-Zitat 2792). Der Umriß ließe sich demnach etwa beschreiben als Ausweitung des Erec-Schemas auf außerhöfische Bereiche (Laudine-Bereich, Märchen, 'Situationsethik'). Damit aber ändern sich auch die gattungsbedingten Grundstrukturen über ihre antinomische Gattungsstruktur hinaus. Iweins zweiter Handlungszyklus ist daher auch weniger eindeutig als der des Erec. ${ }^{4}$ Die Untersuchung der Struktur des Iwein wird sich deshalb mit solchen Grund- und Gattungsstrulkturen beschäftigen müssen. In ihrer Bezüglichkeit auf ein abstrahiertes Artusmodell sind für Hartmann wie für Chrestien die Variation der Aktionen des Helden und die Ausschöpfung der thematischen Verknüpfungen als Textdeutung auf dem Hintergrund der Artusstruktur und der literarischen Situation entscheidend.

Für ein Strukturschema lassen sich Prolog und Kalogrenant-Erzählung als Vorgeschichte deutlich vom ersten Teil trennen. Iweins erster Handlungszyklus reicht zumindest bis zur Heirat mit Laudine; bei der Trennung von erstem und zweitem Tcil ist umstritten, ob die Grenze bei Iweins Heirat, Gaweins Mahnrede, der Anklage Lunetes oder mit Iweins Erwachen gezogen werden soll. Der zweite Handlungszyklus teilt sich kompositorisch in mehrere Abenteuerserien. Alierskampf und Löwenbefreiung sind dabei nochmals vereinzelt. Die übrigen Abenteuer spalten sich in zwei große Gruppen auf, die jede in sich nach dem Prinzip der Schachtelung aufgebaut sind. In die Geschichte von Lunetes Befreiung ist der Kampf Iweins mit dem Riesen Harpin eingeschachtelt, in diesen wiederum die Erzählung des Burgherrn vom Raub Ginovers. Die Zwischeneinkehr bei Laudine und die Auflösung des einsinnigen Erzählstranges durch das Nach-Suchen nach Iwein setzen die letzte Abenteuergruppe ab. In die Rechtshilfe für die jüngere Gräfin vom Schwarzen Dorn und Iweins Kampf mit Gawein ist der Riesenkampf der "Pesme Aventiure" eingeschachtelt, in diesen die Erzählung

${ }^{3}$ Vgl. Hugo Kuhn, "Parzival: Ein Versuch über Mythos, Glaube und Dichtung im Mittelalter," Dichtung und Welt im Mittelalter, 2. Aufl. (1969), S. I69: "programmatische Struktur," und Peter Wapnewski, Hartmann von Aue, 5. Auf. Sammlung Metzler i7 (I972), S. 63: "kompositionelle Strukturen."

${ }^{4}$ Der Iwein gilt ja deshalb als weniger gelungen, vgl. Köhler S. 247. - Kurt Ruh, Hö̈fsche Epik des deutschen Mittelalters: Erster Teil: Von den Anfängen bis zu Hartmann von Aue, Grundlagen der Germanistik 7 (1967), 156. - Wapnewski, S. 72. - Alle Textzitate in Klammern beziehen sich auf folgende Ausgabe: Iwein: Eine Erzäblung von Hartmann von Aue, hrsg. v. G.F.Benecke und K. Lachmann, neu bearb. v. L. Wolff, 7. Ausgabe (1968); alle Hervorhebungen sind von mir. 
der Frauen des Arbeitshauses vom gescheiterten Abenteuer ihres achtzehnjährigen Herrn. Als Schluß und Gegenstück zur Vorgeschichte schließt sich Iweins Zug zum Brunnen und die Versöhnung mit Laudine an.

Schwierigkeiten bietet nicht so sehr eine formale Gliederung, sondern die Beurteilung des Stellenwerts der einzelnen Episoden. Bestimmte Abenteuerserien stehen unter verschieden präzisen Stichworten, die ihre Wertigkeit im Strukturschema bestimmen und sie von den anderen Serien absetzen. Ähnlich ist die Lage bei der Großgliederung, in der erster und zweiter Teil eindeutig unter verschiedenen Norminstanzen stehen. Im ersten Teil ist es Keie unter dem Stichwort von "êre" und "spot," der als Wertenorm für Iwein und die anderen Artusritter erscheint, nur bedingt auch die Königin, die Kalogrenant zum Erzählen auffordert. Keie wird von Iwein an Laudines Brunnen besiegt und verfällt der Schande. An seine Stelle als Norminstanz tritt ausdrücklich Gawein, ${ }^{5}$ der schon durch ein anderes, freundschaftliches Verhältnis zum Helden gekennzeichnet ist. Seine Stichwörter, etwa "trinwe," "rebt" usw. sind nicht so eindeutig festzulegen. Sie hängen von der Interpretation des zweiten Zyklus insgesamt ab. Dazu kommt noch Artus selbst, der als höchste Norm in zweiten Handlungszyklus entschiedener auftritt. Er löst die Königin in dieser Funktion $\mathrm{ab}$ und ist durch seinen ehrenden Besuch bei Laudine und als Schiedsrichter im Gaweinkampf gleichsam die Verkörperung der "coutume."

Schon A. Witte hatte den Iwein in zwei Hauptteile, sog. 'Bücher' aufgeteilt. ${ }^{6}$ Der erste Teil reicht bis zum Ende der Anklage Lunetes (3200) und enthält als Einleitung, Hauptteil und Schluß Kalogrenants Quellenabenteuer, Iweins Gewinn und Verlust von Laudines Liebe. Das zweite Buch hat als Einleitung Iweins Wahnsinn, Löwenrettung und Alierskampf. Der Hauptteil als "Iweins Sühneabenteuer" gliedert sich in die beiden Teile von Lunetes Befreiung und Iweins Kampf mit Gawein. Den Schluß bildet schließlich die Versöhnung. Witte vermerkt wesentliche Unterschiede des Aufbaus im Vergleich mit Chrestien, ${ }^{8}$ die nicht inhaltlich begründet seien. Die Differenzen seien durch verschiedene Aufführungsintentionen verursacht, da man sich fragen müsse, "ob Hartmann überhaupt in demselben Maße wie Kristian an den mündlichen Vortrag des Romans gedacht" ${ }^{9}$ habe.

H. Sparnaay erkennt als Grundlage "das berühmte fünfgliedrige Sche-

${ }^{5}$ Zuerst Hugo Kuhn, "Die Klassik des Rittertums in der Stauferzeit," Annalen der deutschen Literatur: Eine Gemeinschaftsarbeit zahlreicher Fachgelehrter hrsg. v. H.O.Burger, 2. überarb. Aufl. (197I), S. I34.

${ }^{6} \mathrm{~A}$. Witte, "Hartmann von Aue und Kristian von Troyes," $P B B, 53$ (I929), 65-192.

7 Witte, S. 93.

${ }^{8} \mathrm{Vgl}$. das Schema von Witte, S. 87-89.

9 Witte, S. 93 . 
ma"10 der Artusromane, das die originäre Leistung Chrestiens sei. Iweins zweite Abenteuerkette sei eine Art Zusatz zum Prinzip des Erec-Schemas. ${ }^{11}$ Trotzdem bleibe der Artushof "Ausgang und Ruhepunkt der Handlung." 12 Allerdings werde dadurch auch kompositorisch der Iwein nicht wie der Erec nur "die Wiederherstellung der früheren Schuldlosigkeit," sondern der "Aufstieg zum sittlichen Ideal."13

Auf dem Hintergrund der gesamten Artusepik stellt H.Kuhn für den Iwein im Unterschied zum Erec neben dem Artushof ein zweites Zentrum heraus. ${ }^{14}$ Danach stehen dem Helden im ersten Teil Keie, im zweiten Teil dagegen Gawein als programmatische Antagonisten gegenüber. Damit läßt sich auch durch die Struktur das Thema des Romans fassen: "Zuerst Iweins 'êre' gegen Keies Schande; dann Iweins 'triuwe' gegen Gaweins 'êre." "15 Als Zeichen der "triune" Iweins im zweiten Handlungszyklus sieht Kuhn den Löwen "'leo ex macbina,' das Wunder." 16 "In dreimal kunstvoll gesteigertem Einsatz" 17 ist "triuwe" auch das Thema der folgenden Kämpfe Iweins. Unter dem Stichwort der "êre" kontrastieren dazu "drei Termine" als "Hintergrundshandlung" 18 für das 'Versagen' Gaweins. Erst der Zweikampf am Artushof führt diese "Parallelhandlung"19 um Gawein und die Aktionen Iweins wieder zusammen.

E. Köhler deutet die veränderte Struktur des Yvain gegenüber dem Erec diachronisch als Zwischenstufe auf dem Weg zum Perceval. ${ }^{20}$ Auffällig sei mit dem Artushof und der Laudine-Welt die "Nebeneinanderstellung zweier getrennter Lebensbereiche," “die sich nur wie zufällig berühren." 21 Köhlers Strukturschema gliedert den Yvain ${ }^{22}$ deshalb in drei Artushofstationen: Ausgang nach Kalogrenants Erzählung, Lunetes Anklage - dort liegt die Trennungslinie von erstem und zweitem Teil - und dem Zweikampf mit Gavain. Dazwischen staffeln sich die Abenteuer Iweins. Quellenabenteuer, Hochzeit mit Laudine, Gavainrede, Zwischeneinkehr und Versöhnung fallen damit aus der Artus-Abenteuer-Welt.

Im Sinne seiner These von der heilsgeschichtlichen Struktur aller Epen Hartmanns ${ }^{23}$ gliedert W. Ohly Iweins ersten Handlungsteil nach dem Prin-

\footnotetext{
${ }^{10}$ Hendricus Sparnaay, "Hartmanns Iwein," Zur Sprache und Literatur des Mittelalters (196I), S. 223.

${ }^{11}$ Sparnaay, Hartmann von Aue: Studien qu einer Biograpbie, 2 Bände (I 933 und I938), II, 32.

12 Sparnaay, Hartmanns Iwein, S. 223.

${ }^{13}$ Sparnaay, Hartmanns Iwein, S. 226.

${ }^{14} \mathrm{Kuhn}$, "Parzival," S. I68. $\quad{ }^{15} \mathrm{Kuhn,}$ "Parzival," S. I69.

${ }^{16} \mathrm{Kuhn}$, Annalen, S. I $34 . \quad{ }^{17} \mathrm{Kuhn}$, Annalen, S. I34.

${ }^{18}$ Kuhn, "Parzival," S. I69. $\quad{ }^{19}$ Kuhn, "Parzival," S. I69.

${ }^{20} \mathrm{Köhler,} \mathrm{S.} 240 \mathrm{ff} \quad{ }^{21}$ Köhler, S. $247 . \quad{ }^{22}$ Köhler, S. 260.

${ }^{23}$ Walter Ohly, Die heilsgeschichtliche Struktur der Epen Hartmanns von Aue (Diss. Berlin I958), S. 8.
} 
zip zweifacher Schuld (Askalons Ermordung, Terminversäumnis). ${ }^{24}$ Nach dem Wahnsinn seien Iweins Taten ein "Weg der saelde." ${ }^{25}$ Im Anschluß an die Besiegung Harpins sieht Ohly einen Einschnitt: "Aus 'Iwein dem armen' wird der 'riter mittem leun,' der Ritter, der durch das Wunder der triuwe ausgezeichnet ist." ${ }^{26}$ Der Zweikampf mit Gawein stelle schließlich eine "zweite Stufe der Erhöhung Iwcins" 27 dar, nämlich die Wiederanerkennung als höfischer Ritter. Iweins trinnve habe durch die Mächte des Märchens gewirkt; für die schuldige (!) Laudine kann der Erlöste selbst zum Erlöser werden. ${ }^{28}$

Th. Cramer gliedert den zweiten Handlungszyklus ${ }^{29}$ in drei 'Unmachtszustände': Iweins Wahnsinn, seine Ohnmacht an der Quelle und das Nichterkennen durch Laudine. Die beiden letzteren seien durch Hilfsversprechen (an Lunete bzw. die jüngere Gräfin vom Schwarzen Dorn) gekennzeichnet. Der erste Unmachtszustand bildet die Modellgruppe unter dem Thema Land, "vrowne," Recht. Darin sind zwei Befreiungen enthalten, der Alierskampf (mit anschließendem Lohnangebot) und die Befreiung des Löwen. Der zweite Unmachtszustand ist als Durchführung dieses Modells unter den Themen Land und Recht im Bereich der Quelle gedacht. Die beiden Befreiungen sind die Tötung Harpins (Lohnangebot) und die Befreiung Lunetes. Der dritte Unmachtszustand schließlich führt das Thema "vrouwe" und Recht im Bereich des Artushofes durch. Die erste Befreiung ist die der 300 gefangenen Frauen (Lohnangebot), der Zweikampf mit Gawein die zweite Befreiung (!). Jede zweite Befreiung innerhalb der drei Großteile ist dabei ein Kampf für das Recht.

K. Ruh sieht die Abenteuer des Löwenritters "nach der Technilk der Fugung komponiert." $30 \mathrm{Um}$ die Mittelachse der Zwischeneinkehr bei Laudine seien zwei Gerichtskämpfe (Lunetes Befreiung, Gawein-Zweikampf) angeordnet, in die jeweils ein Riesenkampf eingeschachtelt sei. Alierskampf und Löwenbefreiung stünden "außerhalb dieser festen Bindung" als "epische Vorzeichen" 31 für Dankbarkeit bzw. selbstlosen Dienst. Die Ginover-Entführung ist für Ruh ein "technischer Fehler," 32 da sie kein Gegenstück habe..$^{33}$ Die Kompositionsanalyse dürfe sie ausklammern. ${ }^{34}$

\footnotetext{
${ }^{24}$ Ohly, S. I $28 . \quad 25$ Ohly, S. I $29 . \quad{ }^{26}$ Ohly, S. I 29.

${ }^{27}$ Ohly, S. I30. $\quad{ }^{28}$ Ohly, S. I32. $\quad{ }^{29}$ Cramer, S. 42.

${ }^{30}$ Ruh, S. I 53.

${ }^{31}$ Ruh, S. I 53 : "epische Vorzeichen" etwa in dem Sinne, wie sie H. Kuhn für den 'Erec' als "epischen Doppelpunkt" bestimmt hat: vgl. Hugo Kuhn, "Erec," Dichtung und Welt im Mittelalter, 2. Auf. [1969], S. I43.

${ }^{32}$ Ruh, S. I55.

${ }^{33}$ Sie hat ja eins in der Erzählung der gefangenen Frauen der "Pesme Aventiure"!

34 Vgl. das Schema von Ruh, S. I54.
} 
Aus Gründen des "strukturellen Gleichgewichts, das sich sinnfällig in symmetrischen Proportionen ausdrückt" ${ }^{35}$ rechnet H.Linke den Alierskampf und die Löwenbefreiung noch zum ersten Teil. Durch seine Funktion als Gelenkstelle zwischen beiden Teilen erhalte die Löwenbefreiung eine starke "innere Bedeutung," so "daß an der Richtigkeit des Gliederungsansatzes nicht mehr gezweifelt werden kann." 36 Linke nennt denn auch die beiden Teile "Iwein und Laudine" und "der Ritter mit dem Löwen." ${ }^{37}$ Die Werkstruktur ist nach Linke abhängig von der Vortragsgliederung, diese wieder beruht auf Grund der Handschriftenabschnitte auf außerliterarischen Größen wie dem "physischen Leistungsvermögen des Rezitators" und der "Aufnahmefähigkeit des Publikums." ${ }^{8}$ Im Rückschluß von Hartmann auf Chrestien stellt Linke einen Vergleich der Werkstrukturen auf. ${ }^{39}$ Strukturunterschiede werden - über die Vortragsgliederung - letztlich wieder auf die Rezeptionsfähigkeiten eines unterschiedlichen Publikums reduziert. ${ }^{40}$ Der entscheidende Unterschied von Hartmann zu Chrestien sei die Verschiebung der Strukturgrenze. Bei Chrestien bilde sie die Achse zwischen erstem und zweitem Teil, in der Vortragsgliederung gehöre sie eindeutig zum zweiten Teil. Hartmann ziehe sie noch zum ersten Teil. ${ }^{41}$

Den ersten Versuch einer räumlichen Gliederung unternimmt M. Batts. ${ }^{42}$ P. Wapnewski ordnet Iweins zweiten "Aventiure"-Weg "in rhythmisch klare Gruppierungen." "43 Jeweils zwei Befreiungstaten wechseln mit einer Hilfszusage ab. ${ }^{44}$ Darüber hinaus sei noch das Prinzip der Schachtelung und das der Motivdoppelung bzw. -verdreifachung angewendet. ${ }^{45}$ Ein einheitliches Strukturschema entwirft Wapnewski nicht: "Es scheint, als sei die Tektonik des 'Twein' nicht gleichermaßen eindeutig und aussagemächtig wie die des 'Erek'."' 46

\footnotetext{
${ }^{35}$ Hansjürgen Linke, Epische Strukturen in der Dicbtung Hartmanns von Aue: Untersucbungen zur Formkritik, Werkstruktur und Vortragsgliederung (1968), S. 72.

${ }^{36}$ Linke, S. 72.

${ }^{37}$ Vgl. die Tabellen bei Linke, S. 69 und S. $72 \mathrm{f}$.

${ }^{38}$ Linke, S. 79. $\quad{ }^{39}$ Linke, S. 100. $\quad{ }^{40}$ Linke, S. ro5.

${ }^{41}$ Linke, S. I06.- Zu den interpretatorischen Folgen Linke, S. I42 ff: der Löwe als Symbol.

${ }^{42}$ Michael S.Batts, "Hartmanns Humanitas: A New Look at 'Iwein'," Germanic Studies in Honor of E. H. Sebrt (1 968), S.47, der zusätzlich ein Schachtelprinzip, ähnlich dem Wapnewskis, S. 65, hat. Ich gehe auf Batts nicht näher ein, ebensowenig auf den 'tiefenpsychologischen' Ansatz von D.G.Mowatt, "Tristans Mothers and Iweins Daughters," German Life and Letters, 23 (1969/70), I8-3I. ${ }^{43}$ Wapnewski, S. 64.

${ }^{44}$ Nämlich Alierskampf und Löwenbefreiung, Lunetes Tröstung, Harpinkampf und Lunetes Befreiung, Tröstung der jüngeren Gräfin vom Schwarzen Dorn, Befreiung der Frauen und Befreiung (!) der Gräfin im Gawein-Zweikampf. ${ }^{45}$ Wapnewski, S. 65f. ${ }^{46}$ Wapnewski, S. 66.
} 
G. Schweikle stellt neben der Großgliederung in zwei Teile Zweier- und Dreiergruppierungen fest. ${ }^{47}$ Das Doppelungsprinzip sei in der dialektischen Struktur des Romans angelegt. ${ }^{48}$ Zusammenfassend heißt das: "Neben dem offenkundigen Zweierprinzip in der Großgliederung des Werkes scheint für die Binnenstruktur eine Tendenz zur triadischen Ordnung zu bestehen: So in der Kalogrenant-Erzählung, in der ersten und zweiten Bewährungskette, die geschlossene Triaden bilden, oder in der offenen Triade der drei eingeschobenen Erzählungen." 49 Das triadische Prinzip in Verbindung mit bewußter Kontrastierung wirke selbst für Details strukturierend. ${ }^{50}$

Im Unterschied etwa zum Erec fordert ein Strukturschema des Iwein die Einbeziehung zweier Neuerungen: die Trennung in der dialektischen Struktur des Iwein zwischen Artus- und Laudine-Zentrum verlangt die Einbeziehung einer räumlichen Komponente in jedes Schema; zum anderen müssen die Unterschiede zwischen Erzählhandlung und dem in der Erzählhandlung Erzähltem auch im Schema zum Ausdruck kommen.

Das Muster des Brunnenabenteuers wird im ersten Handlungszyklus dreimal verbal und dreimal tatsächlich thematisiert. Kalogrenant setzt mit seiner Erzählung an (93), erzählt sie endlich (259) und hört, wie Ginover sie Artus erzählt (890). Kalogrenants reales Abenteuer wird von Iwein nachvollzogen, schließlich von Artus und seiner Tafelrunde.

Setzt man den Wendepunkt vom ersten zum zweiten Teil nicht auf einen Punkt der 'Schuld' Iweins, sondern an einer strukturell entscheidenden Stelle an, so bietet sich dafür die Erzählerreflexion über die Minne (297I ff). Minne als das Problem des Iwein macht diese Stelle auch thematisch entscheidend. Um diese Erzählerreflexion als einer Art Symmetrieachse entsprechen sich bzw. kontrastieren dann Fristsetzung (280I ff) und Fristübertretung (3029ff), Gaweins Mahnung (2792) und Lunetes Anklage (3 I I I), das Artusfest am Laudinehof (2653) und Iweins Wahnsinn (322 I), Iweins ritterlicher Brunnenschutz gegen Artus (2446) und Iwein als Waldmensch (3261). Iweins Erwachen ist die Zusammenfassung des bisherigen Artuslebens, als handle es sich um einen Traum. Als von Iwein sich selbst erzählte Rekapitulation ist es zugleich der Auftakt zu seinem neuen Abenteuerweg. Wie in der Forschung fast allgemein anerkannt sind Alierskampf und Löwenbefreiung von der übrigen "Aventiure"-Kette isoliert. Diese beiden Abenteuer sind 'epische Vorzeichen' und Hinweise auf die beiden verschachtelten Abenteuerkomplexe: in den beiden vereinzelten "Aventiuren" wird das Grund-

\footnotetext{
47 Günther Schweikle, "Zum Iwein Hartmanns von Aue: Strukturale Korrespondenzen und Oppositionen," Probleme des Erzäblens: Festscbrift für K. Hamburger (1971), S. 6.

${ }^{48}$ Schweikle, S. 7 und vor allem S. I3-17.

${ }^{49}$ Schweikle, S. I3.

${ }^{50}$ Schweikle, S. I6f.
} 
thema der beiden Serien angegeben. Die Befreiung der Damen von Narison geschieht aus Dankbarkeit für die Heilung vom Wahnsinn. Iwein trägt damit eine Schuld ab, handelt aus persönlicher Verpflichtung. Das entspricht der Befreiung Lunetes: Iwein ist an ihrer Lage ja nicht unschuldig, er handelt als persönlich Betroffener (4056f, 4185, 4218f). Der eingeschachtelte Harpinkampf geschieht ebenfalls aus einem persönlichen Beteiligtsein, nämlich aus Freundschaft zu Gawein (4733, 4756ff, 4849ff). Diesem Abenteuerkomplex steht die Ohnmacht am Brunnen voran; auch der NarisonBefreiung geht eine Art 'Ohnmacht' in Form des Wahnsinns voran.

Die Löwenbefreiung schließlich unternimmt Iwein aus ganz anderen Motiven. Der Löwe ist ihm völlig fremd, ebenso wie die jüngere Gräfin vom Schwarzen Dorn und die Frauen der "Pesme Aventiure." Iwein handelt bei der Rettung des Löwen aus einer Mischung zwischen Mitgefühl und Gerechtigkeitssinn. Der Löwe schreit clägelich (3830); darüber hinaus ist der Kampf aber ungleich, da der Drache sich 'unfairer' Mittel bedient (3842f). Der zweite Abenteuerblock unterliegt damit den gleichen Motiven: Iwein handelt sowohl aus Mitgefühl für die versklavten Frauen ("erbermde" 6407, 64I I) als auch aus Gerechtigkeitssinn für die jüngere Gräfin vom Schwarzen Dorn. Dem letzten Handlungsblock geht darüber hinaus - als Hinweis - die Pflege des Löwen nach dessen Verwundung voraus. Daß die Erzählungen vom Ginover-Raub (4526) und vom Abenteuer des Jünglings (6325) sich nicht nur strukturell, sondern auch thematisch (z.B. als Artuskritik) entsprechen, braucht nicht besonders betont $z u$ werden.

$\mathrm{Daß}$ Iwein allmählich zum "Herrn der Zeit" ${ }^{1}$ wird, spiegelt sich in der zunehmenden Komplizierung der Szenenabfolgen, wobei die erzählerische Sukzession von der Szenenreihung (Narison, Löwenbefreiung) zur Verschachtelung (Lunete-Harpin-Lunete) und schließlich zur Mehrsträngigkeit (Nachvollzug von Iweins Weg durch die Botin) übergeht. War Iwein außerhalb jeder Zeit (Wahnsinn, Traum), folgte er dem Schicksal (Löwe), löst er seine Verpflichtungen und kommt dabei in Zeitnot (Lunetes Befreiung): nach der Zwischeneinkehr geht er souverän seinen Weg. Seine Rolle der Zeitbedrängnis übernimmt die Botin im Nachvollzug seines Weges. Der einsinnige Erzähl- und damit Zeitstrang spaltet sich in zwei Stränge auf, die in zeitlicher Verschiebung, aber in sich sukzessiv erzählt werden. Der Harpinkampf (4359) entspricht der Erzählung davon (5831), ebenso Lunetes Befreiung (5145) und der Bericht (5891), desgleichen die Ruhepause auf der Burg (5624) und die Erzählung von dieser Löwenheilung (5956).

Erst nach dem Zusammentreffen der Botin mit Iwein ( 5967 ) ist das Zeitproblem überwunden; Iwein kann trotz Zeitdruck die befreiten Frauen in Ruhe begleiten. Iweins letztes glückliches Abenteuer verbessert endlich die

${ }^{51}$ Ohly, S. I 2 I. 
Muster von Kalogrenants fehlgeschlagenem und seinem eigenen halbgelungenen Versuch.

\section{Strukturschema und Operatoren}

Bei der Untersuchung von 'Operatoren' zur Ausfüllung des Schemas kann es weniger um einen textlichen Vergleich Iwein/Yvain gehen, obwohl auch von der Betrachtung der stilistischen Änderungen Hartmanns Ergebnisse zu erwarten sind. 52 Die Frage nach "Hartmanns dichterischer Selbstauffassung" 53 ist eng verknüpft mit dem Problem mittelhochdeutschen Übersetzens überhaupt. Die Frage müßte deshalb heißen, wie denn die struktur- und bedeutungstragenden Elemente als solche 'Operatoren' auf dem Hintergrund eines abstrahierten Schemas bestimmt werden können. ${ }^{54}$ Das bedeutet wiederum, sie auf ein vergröbertes Schema aufzusetzen. Weniger eine 'Entwicklung' Hartmanns ist daran abzulesen als vielmehr Hartmanns Möglichkeiten, auf diese Weise eine Rezeptionserwartung in einer spezifischen historischen und literarischen Situation gerade noch zu erfüllen. Für den Iwein könnte das etwa bedeuten:

- Wieweit setzt sich die Prologsituation selbst als Norm und überspitzt gleichzeitig diese Situation, z.B. als laudatio temporis acti? Hartmanns Autorbewußtsein würde sich damit auf eine gewisse Routine des Autors in seiner Gattung berufen können. Das Artusmuster wäre so weit eingespielt, daß sich ein Autorrollenspiel mit ihm treiben ließe. ${ }^{55}$ Gaweins Mahnung an Iwein zitiert ja ausdrücklich das 'Erec'-Muster. Wenn der Artusritter Gawein den Artusroman zitiert, wie verhält sich dann der Text Iwein zur Gattung Artusroman? Eine Überspitzung des Typs ins Märchenhafte oder Paradoxe läßt sich beobachten, wenn Minne bis zum Wahnsinn geht, Wundersalben, -ringe und -löwen auftauchen, oder der Erzähler in Exkursen mit sich, dem Publikum, dem Stoff und dem 'Geist der Erzählung' in Widerspruch gerät.

- Eine polare Struktur zeigt sich auch darin, daß ein zweites Zentrum neben dem Artushof besteht. Dieser ist nicht mehr absolut; vielmehr muß er sich zu Laudines Brunnen in Bewegung setzen.

52 Vgl. Witte, S. 72 ff. - Michel Huby, L'adaption des Romans courtois en Allemagne an XIIe et an XIIIe siecle, Publication de la Faculté des Lettres et Sciences Humaines (I 968), S. 95 ff.

${ }^{53}$ Wolfgang Dittmann, "Dune hâst niht wâr, Hartmann: Zum Begriff der wârheit in Hartmanns Iwein," Festschrift für U. Pretzel (1963), S. I I I.

${ }^{54}$ Ernst von Reusner, "Iwein," $D V j s, 46$ (1972), 495.

${ }_{55}$ Vgl. Berufsangabe und 'Dichtungsintention' Hartmanns im Prolog Vers $2 \mathrm{I}-3 \mathrm{O}$. 
- Die Minnethematik reicht vom "universal Christian love-concept of "caritas" " bis zur "even carnal love" 56 und ist nicht mehr untergeordnet oder gleichwertig (vgl. Erec - Enite), sondern im Bild der Minneherrin eng verknüpft mit der Märchenstruktur des Brunnenwunders. Laudines uneindeutige Stellung, ihre sog. Schuld, ihr herrisches Verhalten (vgl. Terminsetzung) und ihr Fußfall gelten ja zumindest als inkonsequent. ${ }^{57}$

- Neben märchenhaft-phantastischen Motiven ${ }^{58}$ dringt außerritterliche, sozial mobilisierende Realität (Waldmensch, Arbeitshaus) ${ }^{59}$ in den Artusroman ein.

- Die Helden verlieren ihre Selbständigkeit im System der "Aventiure;" 60 Märchen und Wunder lösen ihre Aufgaben, ${ }^{61}$ ohne deren Hilfe sind sie 'ohnmächtig.'

- Der Artusroman wird programmatisiert und intellektualisiert. Gegenspieler und -positionen (Kalogrenant/Keie/Gawein) setzen sich ausdrücklich als Alternativen. Präzise Stichworte ("êre," "triune," "kumber," "reht" usw.) deuten auf ein neues Bewußtsein des Helden. Das Abenteuer ist in den Protagonisten verlegt. Iweins Trauer aus Minne ist ein realerer Handlungsanstoß als 'Kausalzwänge' wie z.B. die Zwergenbeleidigung im Erec.

Artusabenteuer und seine Variation

Aus der Stimmung des Artushofes zwischen Spiel und Schlaf ( $65 \mathrm{ff}$ ) entwickelt sich Kalogrenants Erzählung als im Hörerkreis latent vorhandenes Thema : 62

$$
\begin{aligned}
& \text { dise redten von seneder arbeit, } \\
& \text { dise von grozer manbeit ( } 7 \mathrm{I} f) \text {. }
\end{aligned}
$$

Kalogrenants Erzählansatz wird durch Keies Streit um höfisches Verhalten unterbrochen (II3ff). Aber nicht nur als Rückbezug auf Kalogrenants Abenteuer stellt sich der Streit dar, sondern auch als Vorausdeutung auf das Abenteuer Iweins; Iweins erfolgreiche Wiederholung und die Verfolgung Askalons "âne zubt" (1056) sind ja motiviert durch die Angst vor Keies Spott.

${ }^{56}$ H.B. Willson, "Love and Charity in Hartmanns 'Iwein'," Mod. Lang. Rev., 57 (I962), 227.

${ }^{57}$ Zuletzt Reusner, S. $496 \mathrm{ff}$.

${ }_{58}$ Paul Salmon, "The wild man in 'Iwcin' and medieval descriptive technique," Mod. Lang. Rev., 56 (1961), 520.

${ }^{59}$ Z. B.Kuhn, Annalen, S. I 35 : "bewußter Realismus." - Dagegen Sparnaay, Hartmann von Aue, II, 48.

${ }^{60} \mathrm{Köhler}$, S. IOI-IO5.

${ }^{61} \mathrm{Kuhn}$, Annalen, S. 135.- Ohly, S. I42.

${ }^{62}$ Köhler, S. 90. 
Keie fordert Kalogrenant als erster zum Erzählen auf (226f); Ginover muß trotz ihrer Anti-Keie-Position diese Aufforderung aufgreifen ${ }^{63}$ und Kalogrenant selbst zum Erzählen auffordern:

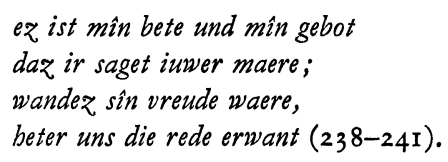

Sie artikuliert damit die Wünsche der Artusrunde und zugleich die der 'real' vorhandenen höfischen Zuhörer Hartmanns. Kalogrenant gehorcht denn auch sofort (243f), nicht ohne, durch Keies Kritik gewarnt, eine Aufforderung zum richtigen Zuhören voranzustellen (245-258). Diese Aufforderung wiederum ist Signal; sie gilt für den Artushof und für Iwein, um beide herum aber auch für den durch die Prologsituation definierten und zur Handlung stimulierten Kreis der höfischen Hörer/Leser.

Der endlich ausgeschlafene Artus, ${ }^{64}$ dem Ginover Kalogrenants mittlerweile eingeschliffenes Handlungs- und Erzählmuster berichtet, will ebenfalls diese "Aventiure" realiter, nicht verbal wie Ginover nachvollziehen. Seine Motivation unterscheidet sich wesentlich von der Iweins. Artus entschließt sich aus "gewonbeit" (893), die er beschwören muß (898). Er setzt einen Termin (900) und will "mit aller sîner mabt" (902) aufbrechen, Iwein dagegen sofort und allein (910). Damit konfrontiert sich Iwein selbst mit Artus und der Tafelrunde. Keie würde mit seiner Schmährede Recht behalten, denn Gawein als dem ersten Vertreter der Artusrunde stünde das Gefecht mit Askalon zu (9I4-9I 8). Will Iwein es "anders" (9I9) machen, so muß er sich aus der für ihn allein repräsentativen Artusöffentlichkeit ausschlieBen. Er verliert damit seinen Status als Ritter der Artusrunde und wird 'freies' Individuum (94If). Damit verliert er aber jede Öffentlichkeit; die erstrebte "êre mit listen" (947) kann als ihrem Wesen nach öffentliche Ehre heimlich gar nicht erworben werden.

Kalogrenants Abenteuer sieht anfangs nach einer definitionsgemäßen "âventiure" aus: unbekanntes Gebiet (26; f), Burg, Inventar, Minnegelegenheit (3 I4) und ein Empfang, wie er sein soll (355f). Doch der Burgherr ist trotz seines idealen höfischen Verhaltens kein Artusritter. Der AventiureBegriff ist ihm völlig fremd:

$$
\begin{aligned}
& \text { und ich bâte geseit } \\
& \text { daz ich nâch âventiure reit, } \\
& \text { des wundert in vil sêre, } \\
& \text { und jach daz im nie mêre }
\end{aligned}
$$

${ }^{63}$ Eva-Maria Carne, Die Frauengestalten bei Hartmann von Aue: Ibre Bedeutung im Aufbau und Gebalt der Epen, Marburger Beiträge zur Germanistik Band 3 I (I 970), S. 37.

${ }^{64}$ Horst Peter Pütz, “Artus-Kritik in Hartmanns 'Iwein'," GRM, 22 (I972), I 94 


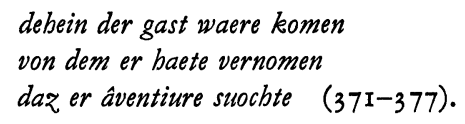

Im zweiten Teil seiner Erzählung verläßt Kalogrenant den höfisch-ritterlichen Raum:

$$
\begin{aligned}
& \text { dan schiet ich unde reit vil vruo } \\
& \text { ze walde von gevilde. } \\
& \text { dâ râmet ich der wilde } \\
& \text { und vant nâch mitten morgen } \\
& \text { in dem walde verborgen } \\
& \text { ein breitez geriute } \\
& \text { ane die liute (396-402). }
\end{aligned}
$$

Sein Zusammentreffen mit dem Waldmenschen (4I 8 ff) ${ }^{65}$ zeichnet sich durch das Fehlen der für den Artusritter 'lebenswichtigen' Öffentlichkeit aus: "âne die liute" (402); allerdings besteht eine Art tierische 'Parodie' auf die Tafelrunde (vgl. 418f und 59f). Dieser 'Freiraum' und das durch seinen Gesprächspartner bestimmte Niveau geben für Kalogrenant die denkbar ungünstigsten Umstände $a b .{ }^{66}$ Hier fallen denn auch die bekannten Selbstdefinitionen beider, "Kennzeichen abgründiger Distanz zwischen dem ritterlichen Menschen und dem Rest der Menschheit." 67 Die Selbstdefinition des Ritters erscheint, wenn sie außerhalb höfischer Gesellschaft und ohne die Vokabeln des ritterlichen Wertesystems vorgetragen wird, simpel und pervertiert. ${ }^{68}$ Der Maxime Kalogrenants (534) entspricht die Aussage des Waldmenschen:

$$
\begin{aligned}
& \text { er sprach'swer mir niene tuot, } \\
& \text { der sol ouch mich ze vriunde bân (484f). }
\end{aligned}
$$

Aus dem Nicht-Verständnis des Waldmenschen für die höfische "Aventiure"-Definition wird ein Mißverständnis, als Kalogrenant ihn auffordert, ihm eine solch definierte "âventiure" zu zeigen (54I). Das zeigt sich an der Repetierung der Definition und ihrer Umdeutung durch den Waldmenschen, wenn dieser "âventiure" mit ungemach gleichsetzt:

$$
\begin{aligned}
& \text { 'sît dîn gemüete stât alsô } \\
& \text { daz dâ nâcb ungemache strebest } \\
& \text { und nibt gerne sanfte lebest, }
\end{aligned}
$$

${ }^{65}$ Vgl. Cramer, S. 35 ff.- Vgl. auch Max Wehrli, "Iweins Erwachen," Formen mittelalterlicher Erzäblung (I969), S. I 89: “Cerberus."

${ }^{66}$ Humphrey Milnes, "The Play of Opposites in 'Iwein'," German Life and Letters, I 4 (I96I), $24 \mathrm{I}$.

${ }^{67}$ Köhler, S. 70 und S. 78 ff.- Gert Kaiser, Textauslegung und gesellschaftliche Selbstdeutung: Versuch einer sozialgescbicbtlichen Interpretation von Hartmanns Artusepen (Diss. masch. Heidelberg, I97I), S. I67 ff.- Ebenso Pütz, S. I95.

${ }^{68}$ Ohly, S. Iosf.- Hugh Sacker, "An Interpretation of Hartmann's 'Iwein'," Germ. Rev., 36 (I96I), 7 f.- Milnes, S. 242. - Batts, S. 39. - Schweikle, S. 8 ff. 
Rolf Selbmann

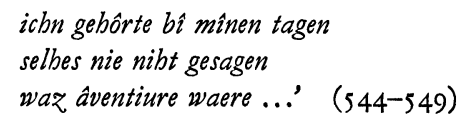

Der Brunnen als dritter und zentraler Teil der Erzählung liegt räumlich genau entgegengesetzt zur Burg: "einen stic ze der winstern bant" ( 599 ; dagegen 265). Als Zweck dieses Abenteuerdranges gilt Kalogrenant der Beweis der "manheit" (632). Im Brunnenfrevel wird sein "suochen" des Abenteuers zum "versuochen" (634).

Mit der gleichen "Aventiure"-Haltung wie Kalogrenant übernimmt Iwein dessen Handlungsmuster und repetiert es. Aus erzähltem Vorwissen variiert er handelnd das Muster: die 'individuelle' Minneidylle Kalogrenants entfällt (978); wegen dieses Vorwissens fällt Iwein beim Unwetter auch nicht in Ohnmacht ( $996 \mathrm{ff})$. Der Nachvollzug des bekannten Musters ${ }^{69}$ erleichtert den Sieg (1004), da Iwein durch scheinbar (!) richtiges Zuhören (vgl. $245 \mathrm{ff}$ ) gegen die Überraschungen gewappnet ist. Er siegt, und damit sprengen Iwein und der Erzähler gemeinsam das Muster auf. In Ermangelung von Zeugen setzt sich der Erzähler zur Wahrheitsinstanz für das Publikum (1033-1046). Statt seiner "des strittes harte vil mit worten" (1029f) erweitert Iwein das Muster" durch die Verfolgung Askalono. Diese Verfolgung "âne zubt" (1056) ist die konsequente Anpassung von Kalogrenants Definition an die veränderte Situation, ${ }^{71} \mathrm{da}$ es sich um Erwerb und Bewahrung seiner "êre" vor dem Spott der Instanz Keie handelt (vgl. 1026-ro66). Der Grund für die Verfolgung, thematisiert unter dem Stichwort der "êre" (I068-107I), gilt der Forschung als Ansatz für die Suche nach der Schuld Iweins. ${ }^{72}$

Als König Artus mit seiner Runde zum Brunnen zieht, versucht Keie das eingeschliffene Handlungsmuster wiederaufzunehmen (2549ff). Doch unter veränderten Kampfbedingungen - denn beide haben den gleichen "Aventiure"-Begriff - unterliegt er gegen den Brunnenherrn Iwein. Keie verfällt der Schande (26r6) und verliert damit seine für Iwein entscheidende Funktion, Instanz des "spot" (2592) zu sein. An seine Stelle tritt Gawein, der im Gegensatz zu Keie in freundschaftlichem Verhältnis zu Iwein steht $(2620$ bis 2623). ${ }^{73}$

${ }^{69} \mathrm{Vgl}$. Ruh, S. 145 .

${ }^{70}$ Sacker, S. Io: Iwein "has to emancipate himself from society."

${ }^{71}$ Pütz meint S. I96, dadurch beweise Iwein seine "Unwürdigkeit." - Ohly, S. I05: "Iweins Wunsch nach einer Trophäe."

${ }^{72}$ Cramer, S. 34f: das Abenteuer sei "aufgesucht," nicht zugefallen wie alle späteren, es werde "für Iwein durch sein Vorwissen zum Willkürakt." - Wapnewski, S. 69, meint, Iwein habe sich des "elementarsten Verstoßes," nämlich "gegen das Recht auf Schutz und Schonung" schuldig gemacht. - Kaiser, S. 172, sieht "einen Zusammenhang zwischen der Erschlagung Askalons und dem Terminversäumnis."

${ }^{73} \mathrm{Kuhn}$, Annalen, S. 133. - Allgemein zum Verhältnis von Keie und Gawein zueinander und zum jeweiligen Helden vgl. Köhler, S. I I I ff. 
Nichtarturische Welt und Minnethematik

Dem unbestrittenen Artuszentrum im Erec steht im Iwein "die Andersartigkeit des Laudine-Reiches mit seiner Eigengesetzlichkeit" "74 gegenüber. Laudines Welt unterscheidet sich von der ritterlichen "Aventiure"-Welt durch wunderbare und märchenhafte Geschehnisse und Dinge: Iwein steckt in einem geheimnisvollen Gefängnis (I I 27f). Wie für Erec bei Koralus ist dieser Ort für Iwein der sozial-materielle Tiefpunkt, aus dem er sich nicht mit eigener Kraft befreien kann. ${ }^{75}$ Statt eines 'realen' Wunders (vgl. Erec: Rüstung und Eriite) hilft ihm ein märchenhaftes: der Zauberring der Lunete (I 202) und ihr Wunderbett (I I 2-I I I 6).

Aus der Sicht des Voyeurs wandelt sich Iweins Zielprojektion. Der Anblick Laudines (I 33 I f) läßt bei ihm urplötzlich Minne ausbrechen. Scheunemann hat darauf hingewiesen, daß damit Minne "das eigentliche Problem" des Romans wird. ${ }^{76}$ Iweins Minneauffassung besteht nun in der völligen Umkehrung seiner bisherigen Anschauungen (1336; vgl. 3009). Damit liegen sein ursprüngliches Ziel (Ehrbeweis vor Keie) und die neue Minne ${ }^{77} \mathrm{im}$ Kampf:

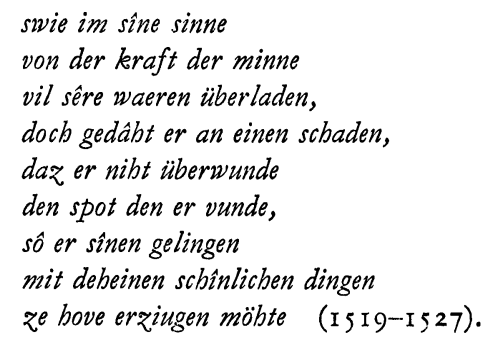

Allmählich gewinnt jedoch die neue Minne die Oberhand ( $536 \mathrm{ff}$ ). Der Erzähler benutzt auch sofort die Gelegenheit zu einer Reflexion über das Wesen der Minne (1557ff). Diese Minne ist Gewalt-Minne, ${ }^{78}$ die Iwein 'umkrempelt' ( 1335 f), also keine Erec-Minne, in der wenig Unterschied in der Wertschätzung zwischen der Rüstung und Enite besteht, und die sich (vorerst) widerspruchslos in die "êre"-Disikussion eingliedern läßt. Der sozialen Gefangenschaft (I I 29) korrespondiert die Minne-Gefangenschaft (I $706 \mathrm{ff}$; vgl. 1737). Immer noch im Zwiespalt zwischen Ehrbeweis und neuer Minne versucht Iwein, beide zu integrieren (1723-1737). Da ihm bisher jeglicher

\footnotetext{
${ }_{74}$ Pütz, S. 196.

${ }^{75} \mathrm{Vgl}$. Ruh, S. I45.

${ }^{76}$ Ernst Scheunemann, Artusloof und Abenteuer: Zeichnung böfischen Daseins in Hartmanns Erec (1937), S. II I.

${ }^{77} \mathrm{Daß}$ es sich nicht um die übliche höfische Minne handelt, hat Reusner, S. $503 \mathrm{f}$ betont.

${ }^{78}$ Aus der Vielzahl der Belege: I424, I 500 . I 5 I 9 f, I 54 6f, I 568, I 656, 2243, 2296.
} 
Ehrbeweis fehlt ( $1587 \mathrm{ff}$ ), sieht er in seinem Streben nach der Gunst Laudines seine Chance, durch Minne seine Ehre zu erweisen (1766-I77I). Er bedenkt dabei nicht, daß das Laudine-Zentrum und Laudine selbst ihrem Wesen nach diesem "êre"-Begriff widersprechen. Strukturell gesehen ist die Heirat der Ausweg, die Artuswürdigkeit wiederherzustellen. Sie erst ermöglicht Iweins Auftreten als Landesherr. Daraus erklärt sich auch, warum Iwein die Quelle gegen Artus schützt: um Artusehre zu beweisen, nicht wie Askalon, und wie Laudine erwartet, um der Quelle willen.

Den Artusrittern scheint der Laudine-Bereich durchaus in ihren Kreis integrierbar. Das Mißverständnis läßt sich an den Prioritäten ablesen, die sie dem Laudine-Bereich zumessen: Iwein z.B. will Laudine, Lunete hingegen sieht Iwein als Landes- und Brunnenherrn:

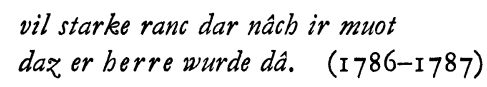

Denn für Lunete und Laudine, das wird aus ihrem Gespräch deutlich, haben "brunnen" und "lant" Priorität vor der "êre" und sind zugleich Voraussetzungen dafür:

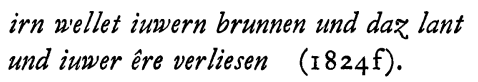

Beschützter Brunnen ist identisch mit bewahrter "êre" (I840-1848; auch 1858, 2935f). "Daß die Umstimmung der Laudine so rasch gelingt, ist weniger die Folge der zweifellos raffinierten Minnedialektik als des Appells der Lunete an die Ehre." "79 Schon hier wird deutlich, daß dieser "êre"-Begriff zweckdienlich auf feste Werte gerichtet und damit ein anderer als der arturische ist. $\mathrm{Daß}$ für Laudine der Land- und Brunnenschutz Bedingung ihrer Ehre ist, wird klar in Iweins Verpflichtung. ${ }^{80}$ Hier ist die Diskrepanz offenbar; Laudine kennt Iwein als Artusritter (2I09f), der "tiurer," d.h. physisch stärker als Askalon ist. Als Individuum ist er uninteressant; ${ }^{81}$ Iwein soll nur die Schutzfunktion übernehmen:

$$
\begin{aligned}
& \text { ern lâze iuch nemen swen ir welt, } \\
& \hat{e} \text { er iu den brunnen bevvar. (2I64f) }
\end{aligned}
$$

Iwein leistet dieses Versprechen im Sinne einer Minnedefinition. Laudine bezieht es auf "mîn abte und mîn guot" (2305).

Den Höhepunkt des ersten Handlungszyklus bildet der Sieg über die bisherige "êre"-Instanz Keie durch Iwein in seiner neuen Beschützerfunktion:

${ }^{79}$ Johannes Erben, "Zu Hartmanns 'Iwein'," ZfdPh, 87 (1968), 349.

${ }^{80}$ Sacker, S. I 3. - Xenja von Ertzdorff, "Spiel der Interpretation: Der Erzähler in Hartmanns Iwein," Festgabe für F. Maurer (I 968), S. I 50 of.

${ }^{81} \mathrm{Am}$ Artushof hingegen war er Lunete aufgefallen, als er sie als einziger grüßte. 


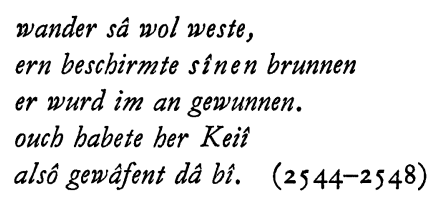

Dabei verteidigt Iwein den Brunnen um den Gewinn von Artusehre gegen den Hof; sein Sieg ist damit ein thematischer. ${ }^{82}$ Aus ihrer Position bleibt Laudine diese Haltung unverständlich; sie nimmt das Faktum des verteidigten Brunnens und den dadurch erreichten Ehrgewinn: "si gedâbte "ich bân wol gevvelt" " (2682). Dieses Mißverständnis bildet offensichtlich den "Keim zu dem Konflikt um die verletzte Ehre." 83

Die Artusöffentlichkeit hatte Iwein beneidet (2648f); seine Eigenschaft als Territorialherr verbindet sich für sie höfisch mit Artusehre. Vom besonderen Wesen Laudines wird nicht gesprochen. Der 'Fehler' des "ungeselle" Gawein (3030) als Gegenfigur des zweiten Handlungszyklus liegt darin, daß er unter den Stichwörtern "bövescheit" (2714) und "êre" (2745) antritt, also mit Artusideologie in einem dem Wesen nach unhöfischen Umkreis. Gawein setzt die Prioritäten anders:

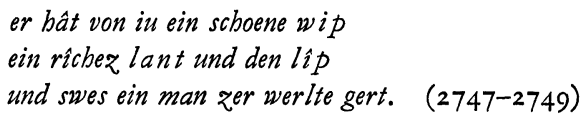

Ritterliche Minne kommt also für Gawein vor der Territorialherrschaft. ${ }^{84}$ Gaweins große Mahnrede ${ }^{85}$ zeigt diese Unterschiede noch deutlicher. Sie findet heimlich (2796) statt. Wieder unter dem Stichwort der "ère" (2774, 2777) erscheint der Erwerb Laudines als ein ritterlicher Akt wie viele andere auch (278 I f). "Iwwer wîhes schoene" steht für Gawein im Vordergrund (2786). Die Ermahnung 'paß auf, daß kein Erec-Roman daraus wird' (2787-2794) zeigt überdeutlich Gaweins Mißverständnis. Es handelt sich ja nicht um ein Erec-Enite-Verhältnis des "ze sêre" Minnens (2798); in der Konsequenz dieses Modells könnte Iwein seine Ehre vielmehr nur dann verlieren (2797), wenn er seine Funktion als Brunnenbeschützer übertriebe, also ' $z u$ sehr' schützte. Gawein indes tut so, als hätte sich für Iwein nichts geändert: "wir suln turnieren als ê" (2803), dabei ist willkürliche Ritterschaft im Sinne der Definition Kalogrenants für Iwein nicht mehr möglich. Das zeigt Vers 3050 ff. Der Zeitvertreib geschieht zwar freudig, doch um so plötzlicher bricht Iweins Reue durch ( $3082 \mathrm{ff}$ ).

Iwcin "versuocht" (2913) Laudine; Laudine ist zunächst enttäuscht, weil sie sich mißverstanden sieht:

\footnotetext{
82 Kuhn, Annalen, S. I34. $\quad{ }^{83}$ Ertzdorff, S. I 52.

84 Das ist um so erstaunlicher, als Gawein selbst ja kein Territorium hat.

85 Vgl. Schweikle, S. I9. - Milnes, S. 242 f.
} 


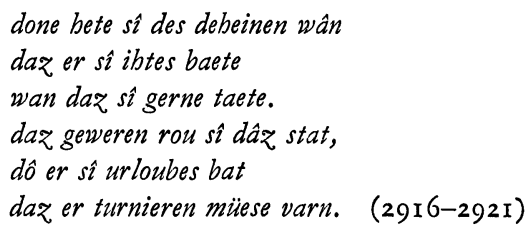

Die Terminfestsetzung ${ }^{86}$ ist daher der Kompromißversuch Laudines, Iweins alte Artusideologie und seine Beschützerfunktion wenigstens auf Zeit zu vereinen. Dieser Versuch muß naturgemäß an der Gegensätzlichkeit beider Welten scheitern. Eine Integration wäre nur möglich, wenn Iwein seine Artushaltung aufgibt und Laudine die ihrige modifiziert. ${ }^{87}$ Der Erzählereinschub zeigt dieses Problem. Der Vorwurf der "vrou Minne" zielt ja darauf, daß beide Welten nicht integriert ${ }^{88}$ sind, daß nur eine Scheinharmonie (2974-2977) besteht. Der Erzähler kann sich nur noch mit dem Verweis auf Quelle und Stoff (2974-2980) und dem Herzenstauschtopos (2990 ff) rechtfertigen. Für ihn ist die Folgc ebenso paradox: einerseits ist Iwein kein Artusritter mehr (3000f), andererseits ein besserer als je zuvor ( $3026 \mathrm{ff}$ ).

Die 'Entwicklung' von Laudines Minne im zweiten Zyklus bleibt rätselhaft. ${ }^{89}$ Ihre Motivation schwankt zwischen 'politischen' Gründen (Brunnenschutz), der Utberzeugungskunst Lunetes und der plötzlichen Minne zu Iwein (234I-2347). Ihr Fußfall schließlich gilt dem Löwentitter, dessen "kumber" sie aus der Zwischeneinkehr kennt und der kein Artusritter mehrist.

Das strukturierende Moment der 'Heimlichkeit' findet sich im Zusammenhang mit der Namensnennung bzw. dem Verschweigen des Namens in der Form von Stellenbelegen ausführlich bei W. Mohr ${ }^{90}$ behandelt. Entscheidende Aktionen jedenfalls finden - im Gegensatz zum Erec - im ersten Teil unöffentlich statt, z.B. Iweins Aufbruch, Ermordung Askalons, Beobachtung Laudines, Gaweins Mahnrede, Waldleben, Wahnsinn usw. Auch darin mag von der Instanz der Artusöffentlichkeit her ein Teil der Bedingungen für die 'Schuld' Iweins liegen. Im zweiten Teil finden entscheidende Szenen immer öffentlich und vor denjenigen statt, die sie betreffen. Auffälligerweise ist es hier Gawein, der seine Teilnahme am Zweikampf verheimlicht und dadurch ins Unrecht gerät (vgl. 5676f). Bei der programmatischen Parallelszene zum Askalonkampf werden die Betroffenen als Zuschauer und Zeugen ausdrücklich hervorgehoben (3723-3727).

${ }^{86}$ Vgl. Ohly, S. I09. - Wapnewski, S. 66: willkürlich aufoktroyierter Termin. Ruh, S. I 5 r. - Ertzdorff, S. 142 f.

${ }^{87} \mathrm{Vgl}$. den Fußfall der Versöhnungsszene.

88 Dittmann, S. I53.

${ }^{89}$ Vgl. Sacker, S. I3. - Sparnaay, "Hartmanns Iwein," S. 21 8. - Carne S. 40. Wehrli, "Strukturen des mittelalterl. Romans," S. 47.

${ }_{90}$ W'olfgang Mohr, "Iweins Wahnsinn: Die Aventüre und ihr 'Sinn'," $Z f d A$, 100 (1971), 86f. 
Situationsethik und neues Bewußtsein

Bekanntlich ist das erste Abenteuer des zweiten Handlungszyklus, der Alierskampf für die Damen von Narison, die Parallelszene zur Verfolgung Askalons "âne zubt." 91 Auch diesmal erreicht Iwein seinen Gegner wie einst Askalon am Burgtor, aber:

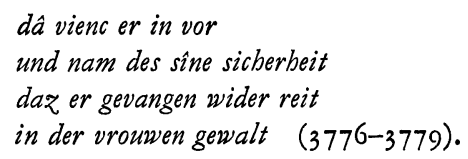

Die dadurch gewonnene Ehre (3785 ff) ist Iweins einziger "lôn" (380I). Die Tat geschah ja, als Signum für alle weiteren Taten der persönlichen Anteilnahme (Lunete, Harpin) aus Dankbarkeit für die ihm erwiesene Hilfe. In dieser Situation findet Iwein den Löwen, als er halb planvoll, halb dem Zufall überlassen weiterreitet:

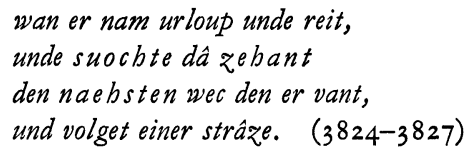

Mit der Befreiung des Löwen ${ }^{92}$ tritt Iwein in ein Stadium der Hilfe für Fremde ein. Auslösendes Moment ist auch hier schon eine Art "erbermde:"

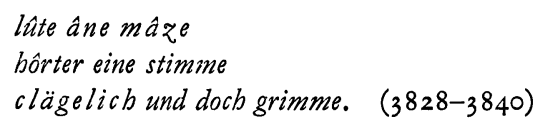

Motiviert ist dieses Eingreifen zugunsten des Löwen nicht nur dadurch, daß dieser das edlere Tier ist (3849), sondern vor allem, weil der Kampf vom Drachen mit unfairen Mittcln geführt wird:

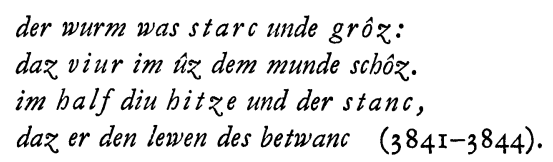

${ }^{21}$ Ohly, S. I I 4. - Ruh, S. I 52. - Sacker, S. I4. - Willson, S. 2 I 8. - Mohr, S. 85. Pütz, S. I $96 f$.

${ }^{22}$ A.T. Hatto, "Der Aventuire Meine in Hartmanns Iwein," Medieval German Studies: Festschrift für F.Norman (1965), S. 96. - Ohly, S. I I6. - Dagegen Sparnaay, Hartmann von Aue, II, 39. - Sacker, S. I 5 f. - Milnes, S. 253 f. - Willson, S. 220. - Besonders ausführlich und mit Forschungreferat Linke, S. I $44 \mathrm{ff}$. - Wehrli, "Iweins Erwachen," S. 186. - Mohr, S. 88f. - Reusner, S. 503. 
Iwein unterstützt also die benachteiligte Partei, die durch diesen Status anscheinend automatisch im Recht ist. ${ }^{93}$ Die Bedeutung des "belfe"-Stichworts kehrt sich später um; das Tier beschämt den Menschen (3869ff) und greift in Iweins Kämpfe trotz aller Hindernisse immer dann ein, wenn dieser einen ungleichen Kampf führt: gegen Übermächtige (Harpin), Ubberzählige (Lunetes Befreiung) oder Übermächtige und zugleich Überzählige ("Pesme Aventiure"). Der Löwe stellt damit die Gerechtigkeit in ungleichen Kämpfen wieder her. Im Zweikampf Mann gegen Mann mit Gawein hingegen wird der Löwe nicht gebraucht und deshalb gar nicht erwähnt. Ferner spiegelt das Verhalten des Löwen den Weg Iweins im ersten Zyklus, wie er hätte sein sollen. Der Löwe wird aus aussichtsloser Lage befreit wie Iwein durch Lunete, wie Iwein vor Laudine unterwirft er sich in Lehensstellung (3869-3871), und beides unter dem Stichwort der Minne (3873). Er übernimmt eine Verpflichtung für Iwein ("Pflege" 3877; vgl. 391 s "buote," dafür gibt es "êre" 3879), so wie Iwein den Brunnenschutz übernimmt und geht sogar eine Art 'Ehevertrag' ein:

und gestuont im zaller sîner nôt,

unz sî beide scbiet der tôt. (388I f)

In diesem Stadium führt der Löwe und Iwein läßt sich den Weg weisen (3918). Das Abenteuer geschieht ihm. Iweins situationsethische Haltung, nämlich aus der Minnetrauer durch "kumber" zu "triwwe" zu gelangen, bestimmt seinen zweiten "Aventiure"-WWeg. ${ }^{94}$

Die Minneohnmacht an Laudines Brunnen bezieht sich exakt auf Iweins Erwachen nach dem Wahnsinn. Dort (3509ff) resümiert Iwein seine Abenteuer des ersten Teils als Traum, allerdings mit Akzentverschiebungen gegenüber dem Bericht des Erzählers. Aus dieser Perspektive thematisiert er selbst seine Aufgabe und seine 'Schuld:'

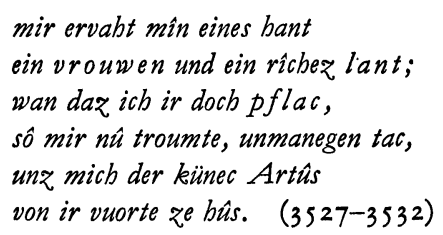

${ }^{93}$ Etwa die jüngere Gräfin vom Schwarzen Dorn, die ihr Recht deshalb bekommt, weil sie von ihrer älteren Schwester übervorteilt worden ist.

${ }_{94}$ Programmatische Äußerungen dazu wären z. B.: 4389 ff, 4432 f, 4509,4855 ff, 5 I I 9 ff, 6072, 6563. - Vgl. auch die Ansätze von Sacker, S. 24f. - Milnes, S. 253 f. Erben, S. 346 und S. 353 f. - Köhler, S. 77 ff. - Batts, S. 45 . - Kaiser, S. 175 ff. Wapnewski, S. 68 f. - Schweikle, S. 20f. - Pütz, S. I97. 
Vers $3547 \mathrm{f}$ ist damit eine Zurücknahme des ersten Artuszyklus: Wer sich an den Traum, d.h. die Werte der Artuswelt hält, gewinnt keine Ehre! Der "gebâre" (3557) ist dabei präziser Bezug auf Gaweins Mahnung vor dem Verbauern. Nach seiner Ohnmacht schließlich (3942) führt Iwein der Treuebeweis des Löwen zur Erkenntnis (395 I ff). Die Monologe (396I und 3509) nehmen die relativierende Artusdiskussion (3969-3978) wieder auf, diesmal in dem Bewußtsein eines realen Erlebnisses. Hier überwiegt eindeutig die neue Instanz der Minne, nicht als höfische "vroide," sondern Minne als Leid, ${ }^{95}$ als "senede swaere" (3982). Aus der Perspektive eines von außen Kommenden und zugleich aus innerer Beteiligung bestimmt Iwein seine Schutzverpflichtung, deren Sinn er jetzt einsieht:



In der darauf bezogenen Wertediskussion (3995-4010) legt er sein neues Programm dar: seine Taten werden Bußtaten seiner Schuld (3999f), bewußt auf eine persönliche Schuld bezogen, ausgelöst durch das Beispiel des Löwen - "rebtiu trinwe" aus "berzeleide" (4001-4005). Folgerichtig trifft er auf die gefangene Lunete unter dem Stichwort des "kumbers" (4029, 4033). Ihre Befreiung ist Bußtat für ihr angetanes Leid (4057), wodurch Iwein geradezu definiert wird:

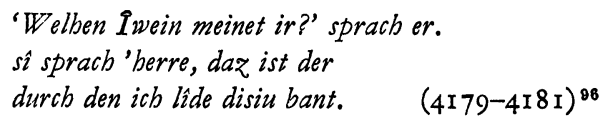

Die Befreiung ist auf Iwein ausgerichtet (4219) und geradezu "heilsnotwendig:" "und swenn ich iu erloeset bân" (4227).

Ein ähnliches persönliches Verhältnis Iweins zu einer der Parteien besteht auch im Kampf gegen Harpin. Der entscheidende Grund für Iweins Eingreifen ist die Hilfe für Verwandte seines Freundes Gawein (4756-4759). Voraussetzung, um die Not überhaupt zu bemerken, ist für Iwein die Erfahrung seines eigenen "kumbers," der ihn für das Leid anderer empfänglich macht:

${ }^{95}$ Daneben ist es ein thematischer Bezug auf Hartmanns Minnesang MSF 218 , 17: "ez ist geminnet, der sich dur die Minne ellenden muoz," wie es in weiterem Zusammenhang der Artusepik Hartmanns und Chrestiens H.Kuhn, "Parzival," S. 172 herausgestellt hat.

${ }^{96}$ vgl. auch "Iwein der arme" (42I3). 
nù erbarmet diz sêre

den rîter der des lewen pflac. (4740f)

Es befähigt ihn zur Frage nach dem Grund für das "trûren" seines Gastgebers $(4432-4440,4507 \mathrm{ff}, 4740 \mathrm{f})$.

Der Konflikt um Iweins doppeltes Hilfsversprechen ist deshalb so schwerwiegend, weil es keinen Kompromiß zwischen den beiden Alternativen zuläßt. Iwein müßte sich entscheiden zwischen religiöser Bußtat und sozialer Rittertat (4854-4858), also letztlich zwischen "Got und ber Gawein" (4864-4868). Die Situation verlangt jedoch Iwein keine Entscheidung ab; denn durch Gottes Hand (49I4f) löst das Auftauchen des Riesen den Gewissenskonflikt.

Erzähltechnisch als Nachtrag (vgl. 5625) wird von der jüngeren Tochter des Grafen vom Schwarzen Dorn berichtet. Ihre Bitte um Hilfe bleibt am Artushof ungehört, da dieser an eigenen Problemen laboriert (Ginoverraub) oder sich schon auf die Seite der anderen Partei geschlagen hat (Gawein). ${ }^{97}$ Die "maere" vom Löwenritter verdichtet sich bis zur stetigen Verfügbarkeit Iweins im Gegensatz zum Versagen der Artusrunde:

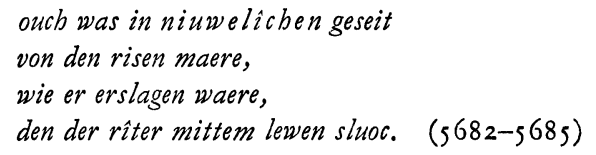

Iwein als anrufbare Instanz wird gesucht, indem seine Abenteuer räumlich nachvollzogen und erzähltechnisch wiederholt werden:

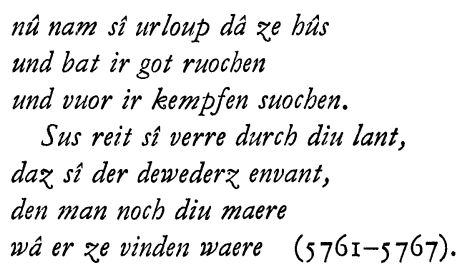

Der Raum zwischen Abenteuer und Laudinezentrum wird inhaltlich ausgefüllt durch die Stichwörter des "kumbers" ( $5785-5788)$ und das Motiv der Namenlosigkeit ( $5726 \mathrm{f}, 5820 \mathrm{ff}$, $5960 \mathrm{ff}$ ). Iweins berühmte Selbstdefinition als Selbstverpflichtung (600I-6004) zum Dienst unabhängig vom sozialen Stand ist die präzise Gegenposition zu Kalogrenants "Aventiure"-Definition.

Die Botin übernimmt wie vorher der Löwe die Führung (6072); Iwein sucht keine Abenteuer mehr, sie fallen ihm zu:

$$
\begin{aligned}
& n \hat{u} \text { sâben sî̀ wâ vor in lac } \\
& \text { ein burg } \hat{u} f \text { ter strâze. (Go8०f) }
\end{aligned}
$$

${ }^{97}$ Kuhn, "Parzival," S. I69. - Schweikle, S. I I. - Pütz, S. I95. 
Aus dieser Haltung heraus kann Iwein die Terminfrage bewußt für seine Zwecke nutzen und die Zeit meistern (6I 56-6I58). Iweins Empfindlichkeit für das Leiden der Frauen (Stichwort "arm") löst wiederum das Fragen aus, bei den richtigen $\left(6_{3} 05\right)$ wie bei den falschen Instanzen $(6236,6265)$. Iwein bietet sofort seinen Dienst an (6300); die Befreiung der Frauen ist ihm Erlösung (6371) durch "erbermde" (6407, 6415): "Die Befreiung der gefangenen und halbverhungerten Seidenweberinnen hat einen betont sozialen Aspekt." 98 Programmatisch setzt Iwein seine Sentenz zur Dienstverpflichtung unter das Signum des 'Schicksals:'

$$
\begin{aligned}
& \text { dir geschibt daz dir gescheben sol, } \\
& \text { und anders nibt, daz weiz ich wol (6567f). }
\end{aligned}
$$

Er geht sogar auf die Bedingung des Riesen ein, da nur durch die Überwindung der "coutume" (6595 ff) die Frauen befreit werden können.

Die eingeschachtelte Erzählung der Frauen von ihrem jugendlichen Landesherrn stellt sich wieder als eine explizite Gegenposition dar. Der Verursacher dieses Unglücks aus "Aventiure"-Leidenschaft steht noch tiefer als Kalogrenant, da er durch seine Niederlage das Unglück anderer verschuldet. Als implizite Kritik am Rittertum erscheint die "âventiure" im Licht eines kindischen Spiels (6328-633I).

Iweins "trinwe" $z u$ Laudine erprobt sich an der Tochter des Burgherrn (6500 ff, 6799ff). Iwein widersteht der märchenhaften und deshalb unguten Idylle (vgl. "Joie de la Curt" im Erec); er agiert souverän in und mit der Zeit (6888I, 6857). Man schreibt ihm schließlich die Prologeigenschaft "saelde und êre" (6864) zu.

Konnte Iwein im außerhöfischen Bereich standhalten, so ist er, um Erfolge zu haben, auf übermenschliche Hilfe angewiesen (Ring, Salbe, Löwe, Gott). Er braucht diese Führer, da seine eigene Kraft nicht ausreicht; er muß sich "fast alle seine Erfolge schenken lassen." ${ }^{99}$ Im Gegensatz dazu kann er den höfischen Zweikampf mit Gawein allein bestehen, da er auf der Seite des Rechts steht. So wird es ein allegorischer Normkampf (7272) um die Wertigkeit der beiden Prinzipien. Iwein siegt moralisch; trotz Reintegration in den Artushof ist dieser nur noch "Durchgangsstation; er ist nicht mehr Ziel, sondern Etappe." 100

\section{Verbalisierte Aventiure und Artuskritik}

Auffällig, etwa wenn man vom Erec ausgeht, ist im Iwein die Verschachtelung der Abenteuer des zweiten Zyklus. Bei der letzten Einschachtelung

\footnotetext{
${ }^{98}$ Köhler, S. 127 und S. 94

${ }^{99}$ Wehrli, "Iweins Erwachen," S. 184. - Ähnlich schon vorher Kuhn, Annalen, S. 135 . 100 Ohly, S. I 23. 
(Ginoverraub, jugendlicher Ritter) handelt es sich um in der Erzählung nachgetragene Handlung in Form von Erzählungen: das Abenteuer wird durch die Erzählung davon ersetzt. Kalogrenants auslösendes Abenteuer ist eine Erzählung; sie fällt in Artus' Pfingstfest, einer traditionellen Erzählsituation: "Man hört 'von grozer manbeit' (72), aber man tut sie nicht." 101 Durch seine Ausführlichkeit, aber auch durch seinen unentschiedenen Ausgang wird der Zweikampf mit Gawein als solcher ad absurdum geführt. An seine Stelle tritt das Wortgefecht, in dem die Entscheidungen in einer paradoxen Lösung liegen. Der verbale Sieger dieses Streites ist zugleich der moralische Verlierer (Gawein! 7577f). Gleiches gilt für Iweins Streit mit Lunete um die Schwere des Leides (4075-4079) und die Diskurse des Erzählers mit "vrou Minne" (z.B. 2979).

Verbale Mißverständnisse (Kalogrenant und der Waldmensch) und ihre Folgen ersetzen tatsächliche Handlungsanstöße. Der Spiel-Raum des Autors erscheint dadurch zwischen Fiktion und Publikumsbezug noch weiter abstrahiert und nur noch in der laudatio-temporis-acti-Erzählung vom König Artus des Prologs konkretisiert. Die Gleichsetzung von "maere" und "werc" (55-58, 93, 432If) ist für Hartmann die Zuspitzung und Zitierbarkeit der eigenen Tradition (Erec-Zitat!) und schließlich die Gratwanderung zwischen autorspezifischen Prinzipien: was wahr ist, ist nur im Erzählen wahr. ${ }^{102}$ Wenn Iweins Abenteuer durch das Suchen der Botin im Nachvollziehen ein Nacherzählen werden, ist die Grenze zwischen Verbalität und spezifischer Faktizität aufgelöst. In solchen Erzählungen von Figuren als Positionen werden die "Aventiuren" frei verfügbar und austauschbar.

Mit geringen Ausnahmen ${ }^{103}$ wird im Iwein die implizite Artuskritik mehr oder weniger anerkannt. Die neuesten Belege bringt der Aufsatz von H.P.Pütz. ${ }^{104}$ Im Prolog zumindest thematisiert das normative und zugleich selektive Ansprechen von Artusrunde und Publikum nur anachronistische Literatur-, Publikums- und Herrschaftsverhältnisse, also Artuskritik in einem sehr weiten Sinn. Hartmanns Iwein erscheint damit als Utberwindung des Artusromans in der Form des Artusromans mit erzählerischen Mitteln. $\mathrm{Daß}$ der Erzähler Artus und seine Runde jeweils ausführlich rechtfertigt, z.B. bei der Ginover-Entführung, bestätigt der Handlungsverlauf jedoch nicht; schon daß es solcher Entschuldigungen bedarf, ist verräterisch. ${ }^{105}$

\footnotetext{
101 Erben, S. 347.

100 Ohly, S. I 23.

${ }^{102}$ Vgl. Dittmann S. I50

${ }_{102} \mathrm{Vgl}$. Dittmann, S. I $50 \mathrm{ff}$.

${ }^{101}$ Erben, S. 347.

${ }^{103}$ Sparnaay, Hartmann von Aue, II, 48. - Ruh, S. Ir ff und S. I 57 ff. - Hans Peter Kramer, Erzäblerbemerkungen und Erzäblerkommentar in Cbréstiens und Hartmanns Erec und Ivvein, Göppinger Arbeiten zur Germanistik 35 (I97I), S. I78f.

${ }_{104}$ Pütz, S. $193 \mathrm{ff}$, ich gehe deshalb nicht näher darauf ein.

${ }^{105}$ Köhler, S. I 80 . - Sacker, S. 5 f. - Dittmann, S. I 55 ff. - Ertzdorff, S. I 56.
} 
Als anachronistisch gewordene "coutume" (5०ff, 34, 893, 5742) ist Artus mit dem Inventar seiner Tafelrunde in seiner ganzen Idealität nur noch Schiedsrichter (734I). Gehandelt wird von exemplarischen Protagonisten, dem konservativen Gawein $(2803,3030-3036)$ und von Iwein, der kein normenkonformer Artusritter mehr ist, sondern selbst neue Normen aufstellt.

Nachträglich sind mir folgende Arbeiten bekannt geworden, die ich leider nicht mehr besprechen kann; sie sollen wenigstens erwähnt werden: Peter Kern, "Interpretation der Erzählung durch Erzählung. Zur Bedeutung von Wiederholung, Variation und Umkehrung in Hartmanns 'Iwein'," ZfdPh, 92 (I973), 338-359. Ders., "Der Roman und seine Rezeption als Gegenstand des Romans. Beobachtungen zum Eingangsteil von Hartmanns Iwein," WW, 23 (I973), 246-252. - Paul Salmon, "âne zuht'. Hartmann von Aue's criticism of Iwein," Mod. Lang. Rev., 69 (I974), 556-56I. 